\title{
Patrimonio mueble urbano de Andalucía
}

Juan Antonio Arenillas Torrejón | Centro de Documentación y Estudios, Instituto Andaluz del Patrimonio Histórico

Luis F. Martínez Montiel | Dpto. Escultura e Historia de las Artes Plásticas,

Universidad de Sevilla

URL de la contribución <www.iaph.es/revistaph/index.php/revistaph/article/view/3402>

\section{RESUMEN}

Un proyecto "Paisaje Histórico Urbano de Sevilla" y el estudio de los monumentos públicos, el mobiliario urbano y el equipamiento de dicha ciudad sirvió como ensayo para definir los objetivos y la metodología de trabajo que más tarde se usó en la formulación del proyecto I+D+i "Patrimonio Mueble Urbano de Andalucía".

Con esta propuesta se pretende confeccionar un instrumento metodológico de acercamiento a los estudios del espacio urbano que proporcione una lectura regional de este tipo de manifestación artística y sirva para regular en alguna medida las actuaciones que en este campo se desarrollan en los diferentes entornos urbanos.

Su difusión contribuirá a una mayor sensibilización y concienciación de la ciudadanía sobre este tipo de patrimonio, y al establecimiento de medidas para su conservación y protección.

\section{Palabras clave}

Patrimonio mueble urbano | Mobiliario urbano | Espacios urbanos | Monumentos | Proyectos | Ciudades | Calles | Paisaje cultural | Esculturas conmemotativas 


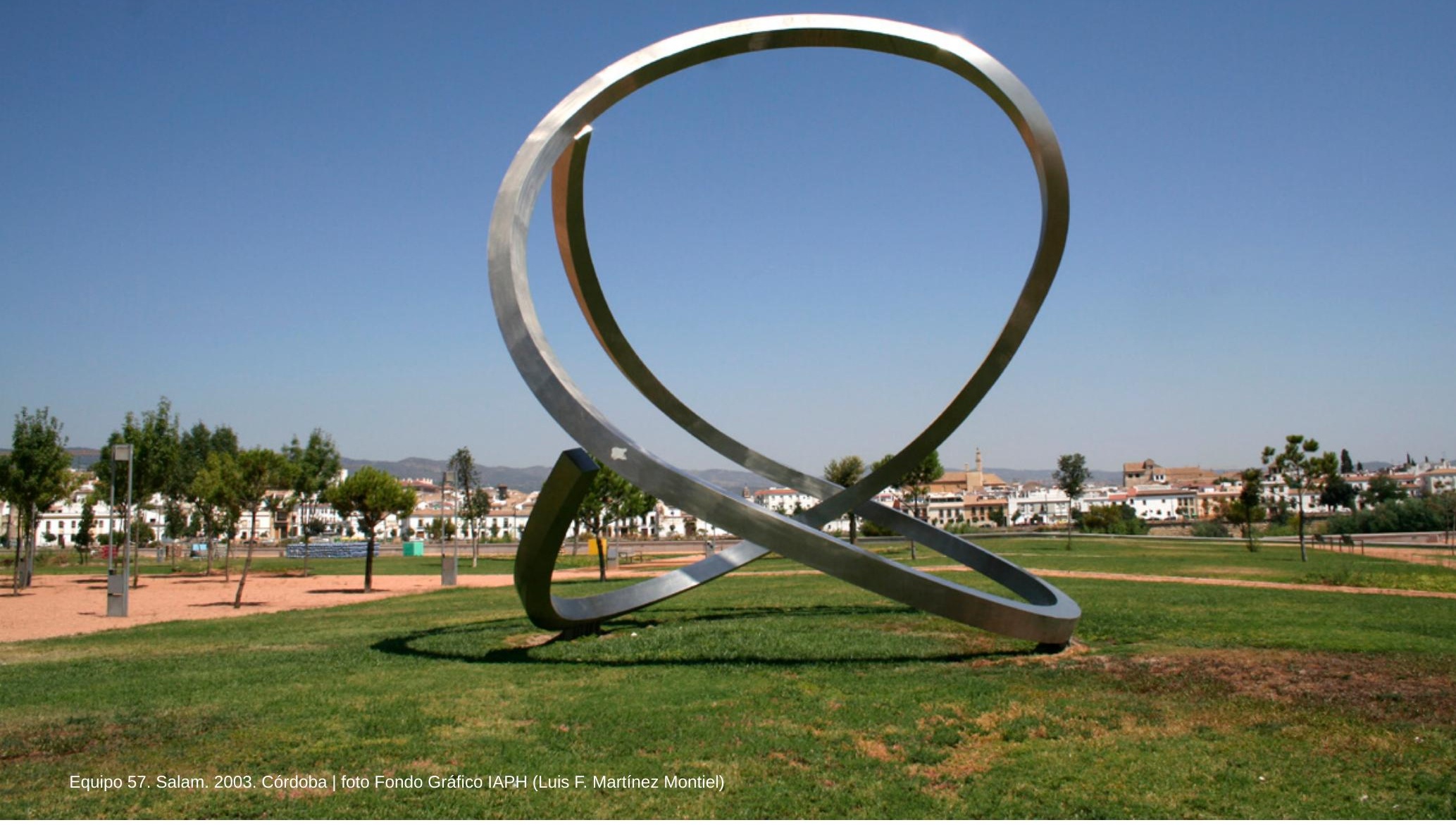


1

Algunos de los estudios fueron publicados en PAISAJE, 2011.

2

En la ciudad de Cádiz se inician los trabajos en 2011, colaborando un equipo conformado por profesionales de las universidades de Cádiz y Sevilla y del IAPH. En Almería, Granada, Huelva y Málaga, en 2012 y en Córdoba y Jaén se espera hacerlo en 2013.

3

El equipo está compuesto por veinticuatro investigadores de los cuales dieciocho son historiadores del arte pertenecientes a distintas universidades andaluzas y seis, al $\mathrm{IAPH}$. Abarcan las disciplinas de historia de arte, antropología, arqueología, geografía, fotografía y documentación.
En 2010, en el marco del proyecto "Paisaje Histórico Urbano en Ciudades Históricas Patrimonio Mundial: Indicadores para su Conservación y Gestión", en el que el Instituto Andaluz del Patrimonio Histórico (IAPH) colabora con el Centro de Patrimonio Mundial de la UNESCO, se encuadra la propuesta "Paisaje Histórico Urbano de Sevilla". De esta última deriva el trabajo inédito denominado "La construcción del espacio urbano: mobiliario y equipamiento", ejecutado entre 2010 y 2011, en el que se abordó el estudio y análisis de los monumentos públicos, el mobiliario urbano y el equipamiento de dicha ciudad, es decir, su patrimonio mueble urbano, elaborándose para ello una nueva y novedosa metodología de trabajo que se viene aplicando en el territorio andaluz ${ }^{1}$.

Para dicho trabajo, el Centro de Documentación y Estudios del IAPH contaba con referentes tan significativos como la Guía del Paisaje Cultural de la Ensenada de Bolonia o el Registro de Paisajes de Interés Cultural de Andalucía, experiencias en las que la documentación y estudio del patrimonio cultural trascendía al objeto individual y aislado mediante el establecimiento de relaciones espaciales y formales de cada objeto mueble con valores patrimoniales, con la ciudad y con otros objetos (GUÍA, 2004; RODRIGO CÁMARA; DÍAZ IGLESIAS; FERNÁNDEZ CACHO et ál., 2012).

En 2011, la labor desempeñada y la metodología empleada en la ciudad de Sevilla sirvieron como base para la formulación del proyecto "Patrimonio Mueble Urbano de Andalucía", previsto para el período 2011-2013, que pretendía abarcar las capitales de provincia y las ciudades medias. Se firman entonces convenios específicos de colaboración con las universidades de Cádiz, Granada y Málaga para el estudio y análisis de los monumentos públicos y mobiliario urbano. Un año más tarde la Universidad de Almería se incorpora al proyecto ${ }^{2}$. En el caso de la ciudad de Huelva, los trabajos serán llevados a cabo por un equipo conformado por profesionales del IAPH y de la Universidad de Sevilla.

El proyecto fue presentado por el IAPH a la convocatoria del Ministerio de Economía y Competitividad, Plan Nacional de I+D+i (2008-2011), subprograma de proyectos de investigación fundamental no orientada, convocatoria 2012. Su concesión definitiva se ha producido en junio de 2013 y se completará entre los años 2013 y $2015^{3}$.

Se partió, desde el principio, de considerar que las ciudades son el fruto de las acciones y del pensamiento de todos aquellos que viven y vivieron en ellas; espacios construidos y espacios vacíos y, entre ambos, la memoria forjando la ciudad. Una ciudad es lo visto y lo imaginado, lo real y lo soñado, lo sentido y lo deseado. Una ciudad nunca es sólo una ciudad. Una ciudad es tantas como imágenes produzca en los que la viven, los que la desearían 

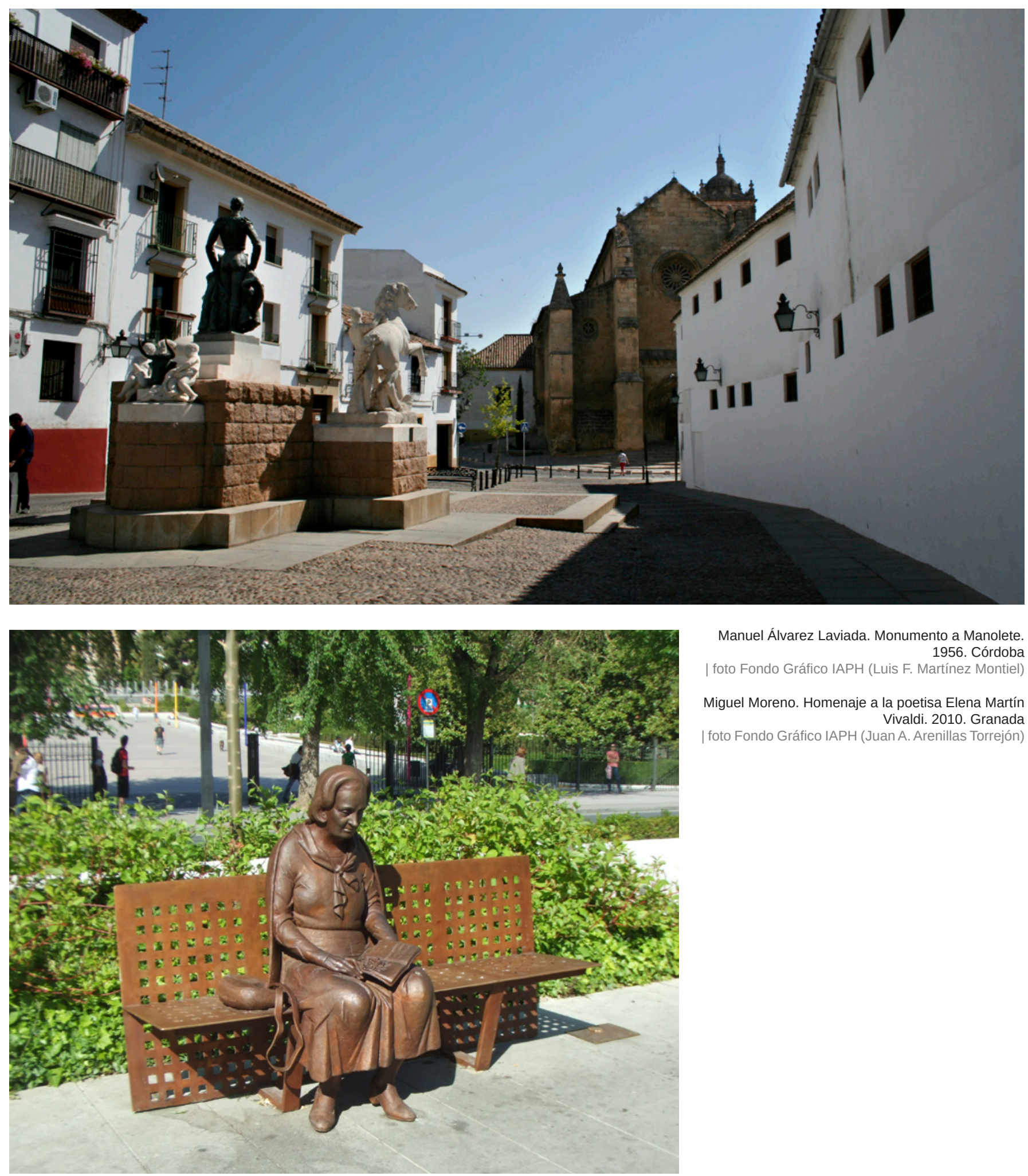

Manuel Álvarez Laviada. Monumento a Manolete. 1956. Córdoba | foto Fondo Gráfico IAPH (Luis F. Martínez Montiel) Miguel Moreno. Homenaje a la poetisa Elena Martín Vivaldi. 2010. Granada | foto Fondo Gráfico IAPH (Juan A. Arenillas Torrejón) 


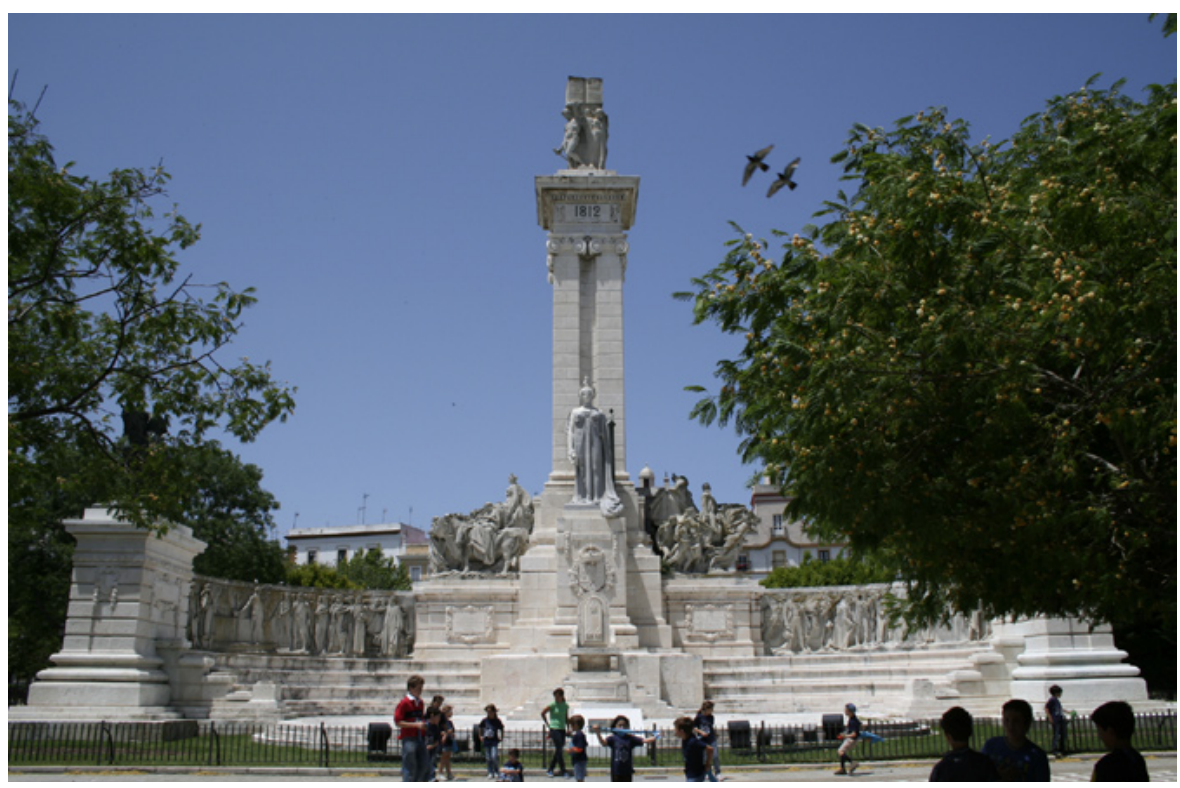

Modesto López Otero (arquitecto) y Aniceto Marinas y García (escultor). Monumento a las Cortes de Cádiz. 1911-1929. Cádiz | foto Fondo Gráfico IAPH (Luis F. Martínez Montiel)

vivir y los que la vivirán. En definitiva, una ciudad es la suma de todas las acciones y percepciones que sobre ella se acometan y tengan.

En ese sentido, trabajar con los monumentos públicos de una ciudad supone intentar clarificar cuáles son las grandes corrientes por las que esa ciudad es y se significa. Acercarse a sus monumentos implica desenmarañar toda una trama de opiniones que, a veces de una forma contradictoria, han ido formando cada una de sus realidades. Dos condicionantes, el tiempo y el espacio, serán primordiales para el análisis del patrimonio mueble urbano andaluz. Evidentemente los periodos históricos en que surgieron los monumentos resultan básicos a la hora de entenderlos, pero no lo es menos el espacio para el que fueron concebidos. Así pues el juego con estas dos variables se ha convertido en el hilo conductor del presente proyecto y en el inspirador para establecer relaciones que, como todas aquellas que nacen de la subjetividad, pueden ser puestas en entredicho por subjetividades diferentes.

Los procesos históricos, las actividades socioeconómicas, serán claves para el estudio a nivel regional. Del análisis general a nivel territorial, se podrá descender al nivel de cada ciudad en sus caracteres diferenciadores. Hechos como el descubrimiento de América pueden ofrecer un análisis a nivel regional con múltiples matices y manifestaciones, pero también pueden ser determinantes, a nivel de la ciudad, acontecimientos o procesos históricos concretos como la Reconquista o la Constitución de 1812, el encuentro de culturas, actividades como la minería y la industria, o manifestaciones 


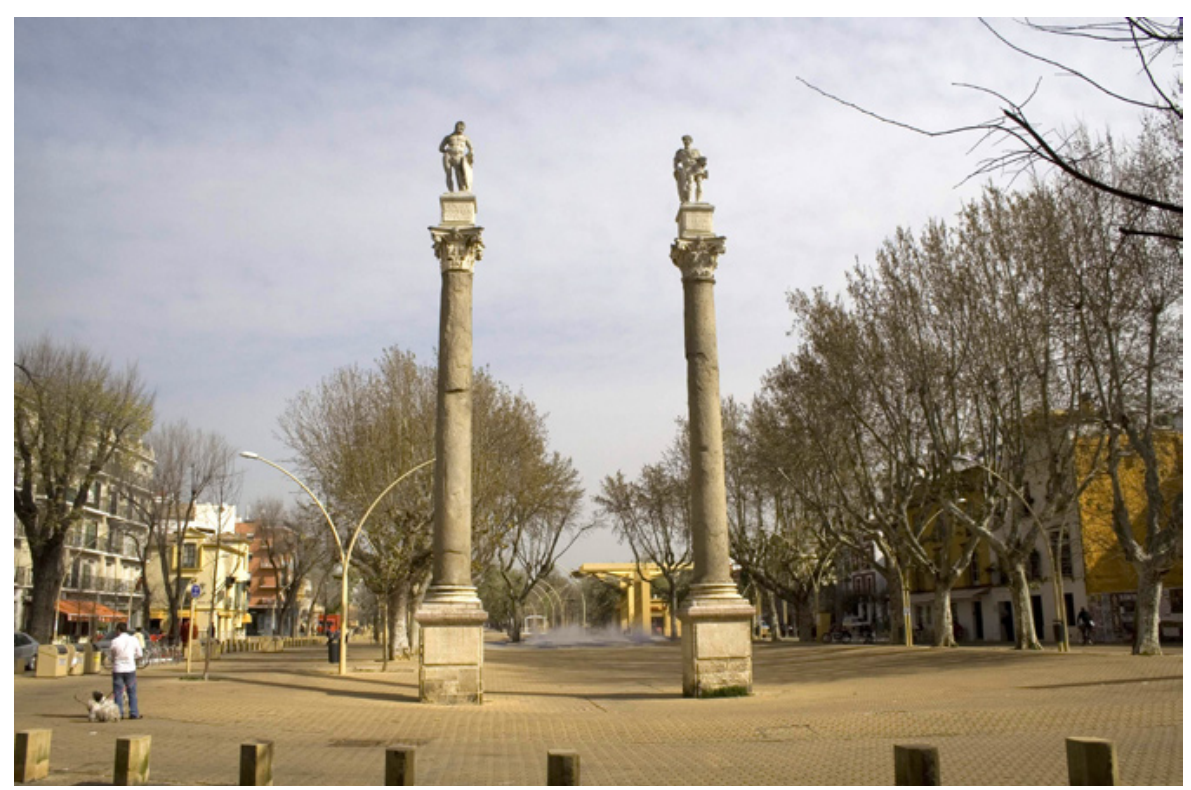

Diego de Pesquera. Columnas de Hércules y César. 1574. Sevilla | foto Fondo Gráfico IAPH (Luis F. Martínez Montiel)

contemporáneas como el grafiti ${ }^{4}$. Todos ellos conformarán y diferenciarán a las distintas ciudades, singularizándolas dentro de la cada vez más devastadora globalización.

En lo que se refiere a una bibliografía especializada, puede afirmarse que escasean los estudios dedicados a este tipo de patrimonio. No en vano, los primeros dedicados a esta manifestación artística corresponden a la década de los 80 del siglo XX, sobre las ciudades de Cáceres y Barcelona (LOZANO BARTOLOZZI, 1988; SUBIRACHS I BURGAYA, 1989). De mayor interés resulta la investigación que aborda la escultura pública en Valencia, en la que su autora contempla el contexto histórico, los criterios estéticos de la época y el espacio urbano como los condicionantes básicos usados en su estudio (HERAS ESTEBAN, 2003). Se cuenta también con obras más genéricas que relacionan esta manifestación pública con aspectos como la historia, la política o el paisaje. En estos aspectos la publicación de Carlos Reyero (1999) puede ser considerada como clave en cuanto a escultura conmemorativa. En Andalucía, especialmente interesante por la amplitud de sus argumentos, debe reseñarse la monografía El Monumento Público en Sevilla de Espiau Eizaguirre (1983). En ella no sólo se analizan los monumentos, las fuentes y las cruces o cruceros, sino que se ofrece una visión de este patrimonio relacionado con el espacio urbano. Asimismo, se deben resaltar la obra Sevilla Turística y Cultural. Fuentes y Monumentos Públicos, de Lafita Gordillo (1998), y los estudios realizados por Gabriel García para Granada y por Juan Antonio Sánchez para la ciudad de Málaga (GARCÍA GUARDIA, 2002; SÁNCHEZ LÓPEZ, 2007; SÁNCHEZ LÓPEZ, 2005).
Para el caso de Sevilla se definieron cinco categorías o líneas argumentales: El fenómeno americano: consecuencias y memoria; Desastres; Glorificación: la ciudad piensa en sí misma; La Exposición Iberoamericana de 1929; y La Exposición Universal de 1992. Tras esta primera fase del estudio, se viene trabajando en otras como el comercio, la religiosidad o sacralización del espacio público, etc. 


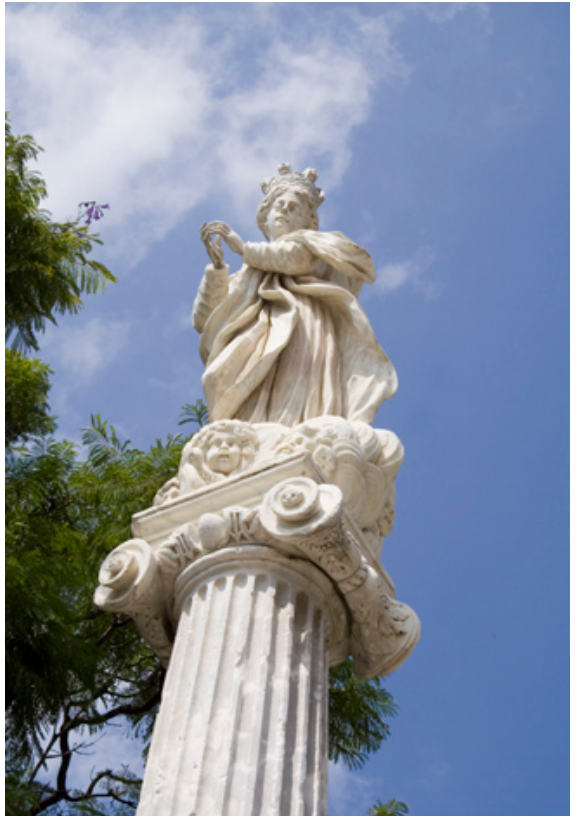

Anónimo. Triunfo de la Inmaculada. 1695. Cádiz | foto Fondo Gráfico IAPH (Luis F. Martínez Montiel)

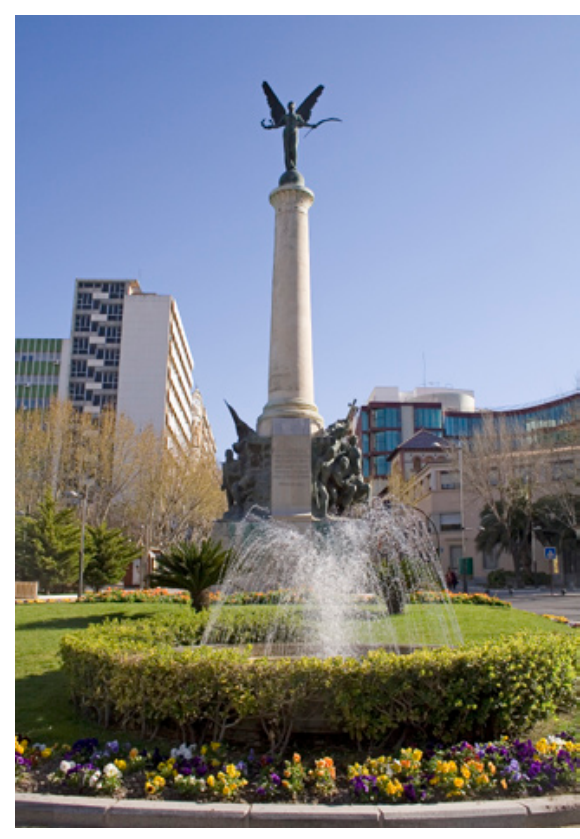

Jacinto Higueras. Monumento a las Batallas. 1910. Jaén | foto Fondo Gráfico IAPH (Luis F. Martínez Montiel)

Fuera del ámbito español existen interesantes estudios sobre mobiliario urbano, destacando los de Seixas (1993) o Bebiano (1995) para la ciudad de Lisboa, o el de las francesas Boyer y Rojat-Lefebvre, Aménager les espaces publies: le mobilier urbain (1994).

A las publicaciones citadas debe añadirse la de Silvia Segarra, Mobiliario Urbano: historia y proyectos (2012), en la que cobra especial significación el mobiliario urbano industrial de los siglos XIX y XX.

Si a la escasez de estudios se une la práctica inexistencia de ordenanzas públicas que regulen este patrimonio, los trabajos previstos en este proyecto, cuyo objetivo final es la posibilidad de alcanzar resultados prácticos, se pueden convertir en un instrumento clave para el futuro.

\section{OBJETIVOS}

El objetivo general del proyecto es el de confeccionar un instrumento metodológico de acercamiento a los estudios del espacio urbano desde la perspectiva de los monumentos públicos, proporcionando una lectura regional de este tipo de manifestación artística, de la que se carece totalmente en la actualidad. Con este se intenta trabajar en los elementos que lo componen como factores básicos y que contribuyen al enriquecimiento de la ciudad y a la percepción que de ella se tiene. Asimismo, y aunque inicialmente a nivel 
puramente teórico, uno de sus principales objetivos es el de dotar de un instrumento de carácter científico, que sirva de base para regular en alguna medida las actuaciones que en este campo se desarrollan en los diferentes entornos urbanos.

Para alcanzar esos objetivos se exige el conocimiento del patrimonio mueble urbano de cada ciudad con su identificación en distintas categorías y la elaboración de su registro. El análisis de cada uno de los objetos teniendo en cuenta el contexto histórico, el espacio urbano y los criterios estéticos, entre otros, llevará a la formulación de recomendaciones para las nuevas creaciones, así como para las actuaciones de conservación del patrimonio mueble urbano. Al mismo tiempo, este análisis puede constituir un primer paso para el impulso de instrumentos que permitan a los ayuntamientos de la comunidad andaluza un mayor conocimiento sobre su patrimonio mueble urbano y unas mejores y más efectivas bases en las que apoyar sus actuaciones en este campo. Lograr que la ciudadanía se sienta identificada y sensibilizada hacia este tipo de patrimonio, contribuirá a su mejor protección y conservación y a dotar de mayor significado para la ciudad cualquier propuesta sobre nuevas aportaciones a su patrimonio. De esta forma y, en alguna medida, se podrá hacer frente a la continua banalización de los espacios urbanos que se viene sufriendo últimamente. No es este un proyecto alejado de la realidad en el que se intente valorar asépticamente sino que, antes bien, pretende establecer una serie de claves para que las actuaciones que finalmente se lleven a cabo sean más certeras y asuman los errores cometidos. En casi todas las ciudades andaluzas y en muchas españolas y europeas se ha producido, en los últimos años, una "muñecalización", en el peor de los sentidos, del espacio urbano. La escasez de piezas de interés -no sólo por su evidente falta de calidad artística y estética sino por la lejanía de las tendencias creativas más valoradas en la actualidad, así como la intrascendencia de los hechos o personajes- es tan alarmante que a veces parece que las ciudades sufren ataques internos para autodestruir la reputación conseguida. También este escaso nivel afecta a las creaciones pasadas. No se trata de sobrevalorar las creaciones de épocas precedentes, sino de poder establecer una serie de criterios mínimos que sirvan para dignificar los monumentos de nuestros espacios urbanos, aunque ello conlleve, en no pocas ocasiones, su retirada.

\section{METODOLOGÍA DEL PROYECTO}

La metodología propuesta para este proyecto hereda formas de proceder habituales del IAPH. Por tanto, con objeto de ofrecer un diagnóstico analítico sobre el papel del patrimonio mueble urbano, así como proponer criterios que incidan en las nuevas intervenciones, el proyecto plantea desde los inicios el establecimiento de una clara metodología de trabajo, que permita 
5

Un primer ensayo de esta metodología ha sido contrastada en el caso de la ciudad de Sevilla. La continuación del análisis en la ciudad hispalense así como el inicio del proceso de trabajo en el resto de ciudades está contribuyendo a una mejora de la metodología. un acercamiento global a todos aquellos bienes muebles de carácter urbano monumentales y al equipamiento que en numerosas ocasiones acompaña a éste en la construcción del espacio ciudadano ${ }^{5}$.

El método de trabajo plantea establecer una serie de fases, a veces coincidentes en el tiempo, que marcarán su desarrollo. Básicamente se propone un análisis del estado de la cuestión para, en virtud del diagnóstico, programar una serie de recomendaciones tendentes a la mejora en cuanto a valoración, conservación y disfrute del patrimonio, sin olvidar las ricas posibilidades que un estudio de este tipo puede producir en las nuevas creaciones.

Asumiendo que el faseado puede ir perfilándose y definiéndose durante el proceso de trabajo, inicialmente se proponen las siguientes etapas:

\section{1. ${ }^{a}$ Fase. Selección documental y selección de patrimonio mueble urbano}

Se procederá a una búsqueda de fuentes bibliográficas, documentales y gráficas para conocer el estado de la cuestión. Tras la consulta bibliográfica se pasará a confeccionar un primer listado con los elementos referentes en el estudio. Se pueden establecer dos tipologías: mobiliario monumental y equipamiento. En el primer grupo se incluyen aquellos que aportan valores básicamente estéticos y ornamentales a la ciudad: esculturas monumentales, fuentes, cruceros o cruces y, para completarla, paneles conmemorativos. En la segunda categoría, se recogerían bancos, farolas, fuentes de abastecimientos, marquesinas, etc.

El análisis de esos listados tipológicos y el conocimiento de los acontecimientos o procesos históricos y las actividades socioeconómicas de cada una de las ciudades permitirán ir estableciendo líneas argumentales con sus correspondientes entidades o recursos patrimoniales.

\section{2. ${ }^{a}$ Fase. Realización de trabajo de campo}

A partir de esos listados se inician las primeras visitas para llevar a cabo las tareas relacionadas con el trabajo de campo. De los monumentos escultóricos, fuentes, cruceros, placas conmemorativas y todo lo referente a equipamiento, se seleccionarán aquellos elementos que tipológicamente resulten más significativos y frecuentes, pues -como se verá más adelante- uno de los grandes problemas del equipamiento en la ciudad es su enorme diversidad y, en muchos casos, la reiteración de modelos estándares en ciudades del entorno y, a veces incluso, nacionales. No resulta extraño observar cómo los mismos modelos se reparten por toda la geografía autonómica y nacional. 

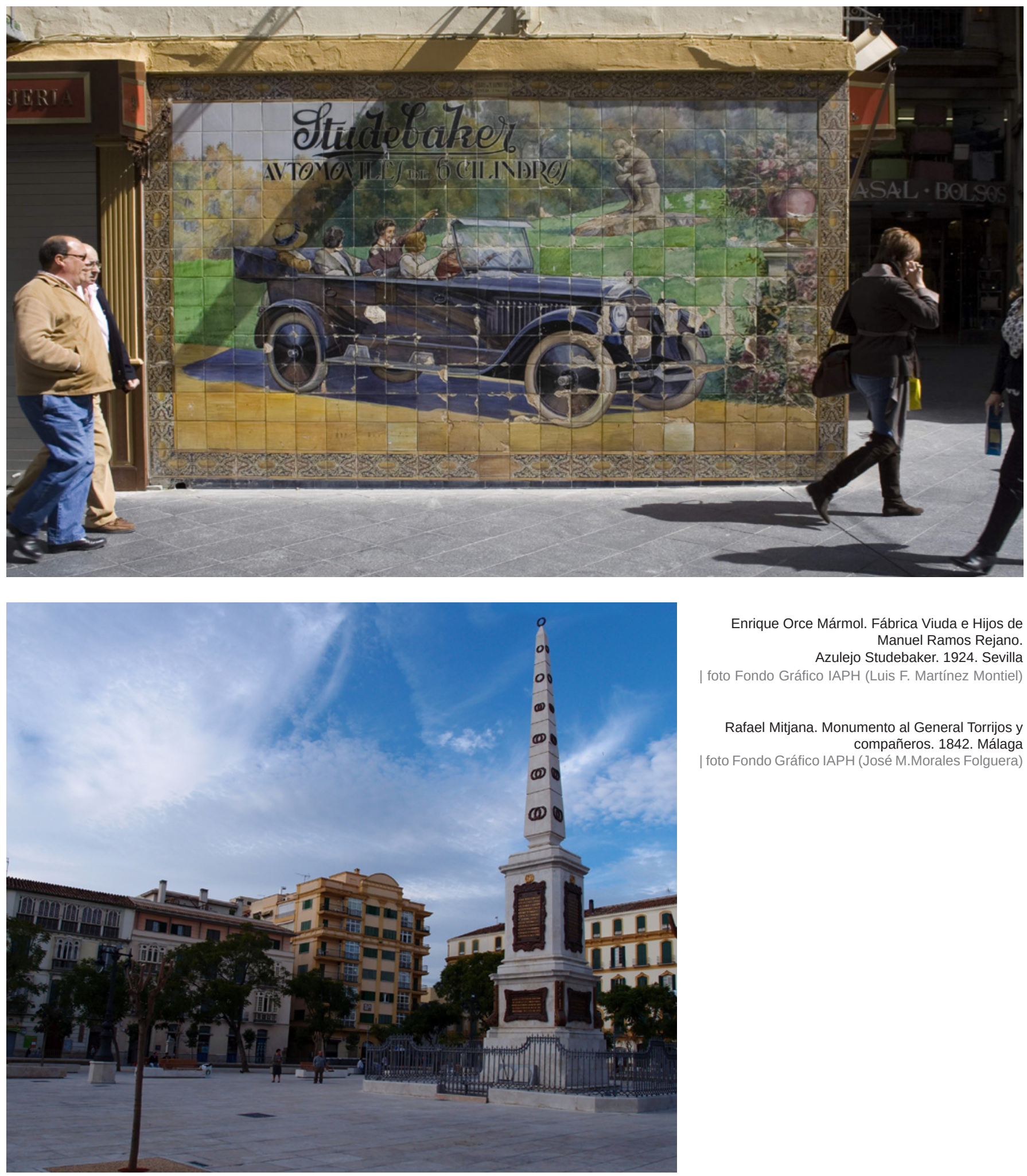

Enrique Orce Mármol. Fábrica Viuda e Hijos de Manuel Ramos Rejano Azulejo Studebaker. 1924. Sevilla | foto Fondo Gráfico IAPH (Luis F. Martínez Montiel)

Rafael Mitjana. Monumento al General Torrijos y compañeros. 1842. Málaga | foto Fondo Gráfico IAPH (José M.Morales Folguera) 


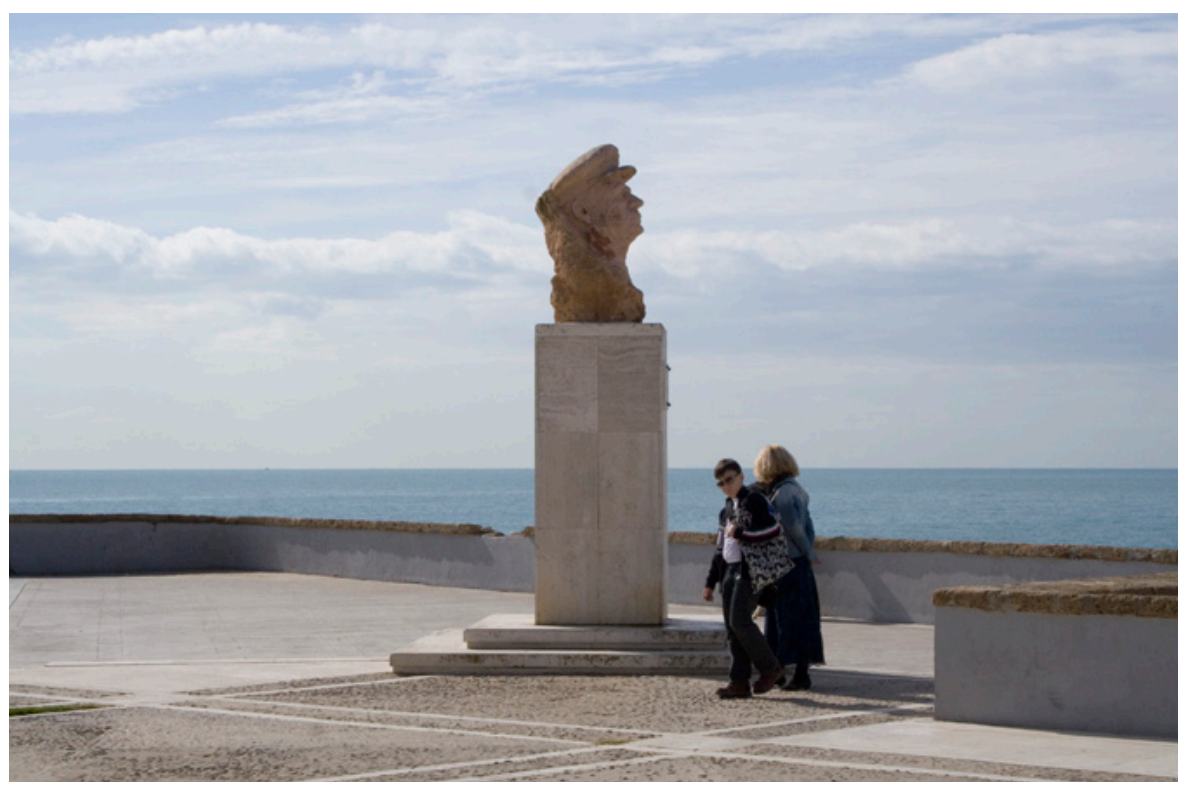

Fernando Benítez. Monumento a Paco Alba. 1979. Cádiz | foto Fondo Gráfico IAPH (Luis F. Martínez Montiel)

La documentación gráfica adquiere un papel de gran relevancia puesto que debe recogerse no sólo la trascendencia individual del objeto, sino también su significado espacial y su relación con la ciudad. Ello debe plantear desde el primer momento la realización de un variado número de tomas que sea capaz de convertirse a posteriori en un importante material de estudio para los diferentes tipos de análisis a los que se verán sometidos.

Se utilizará un modelo concreto de ficha de trabajo, ya contrastado en el caso de Sevilla, para incorporar todos los datos durante la fase de los trabajos de campo y los necesarios en la fase de estudio. Esta deberá permitir establecer parámetros claros para recoger la información y permitir su posterior análisis.

\section{3. ${ }^{a}$ Fase. Ficha síntesis para la recogida de información (líneas} argumentales, referenciación geográfica, criterios de valoración)

La ficha de las líneas argumentales recoge información que se agrupa en cuatro módulos:

1. Identificación y descripción: información sobre denominación, localización, delimitación, planimetría e imágenes.

2. Descripción y análisis: listado de los recursos patrimoniales incluidos, así como sus cualidades y valores.

3. Valoración crítica y diagnóstico, en función del análisis de los indicadores de valoración de los recursos patrimoniales.

4. Criterios y recomendaciones 


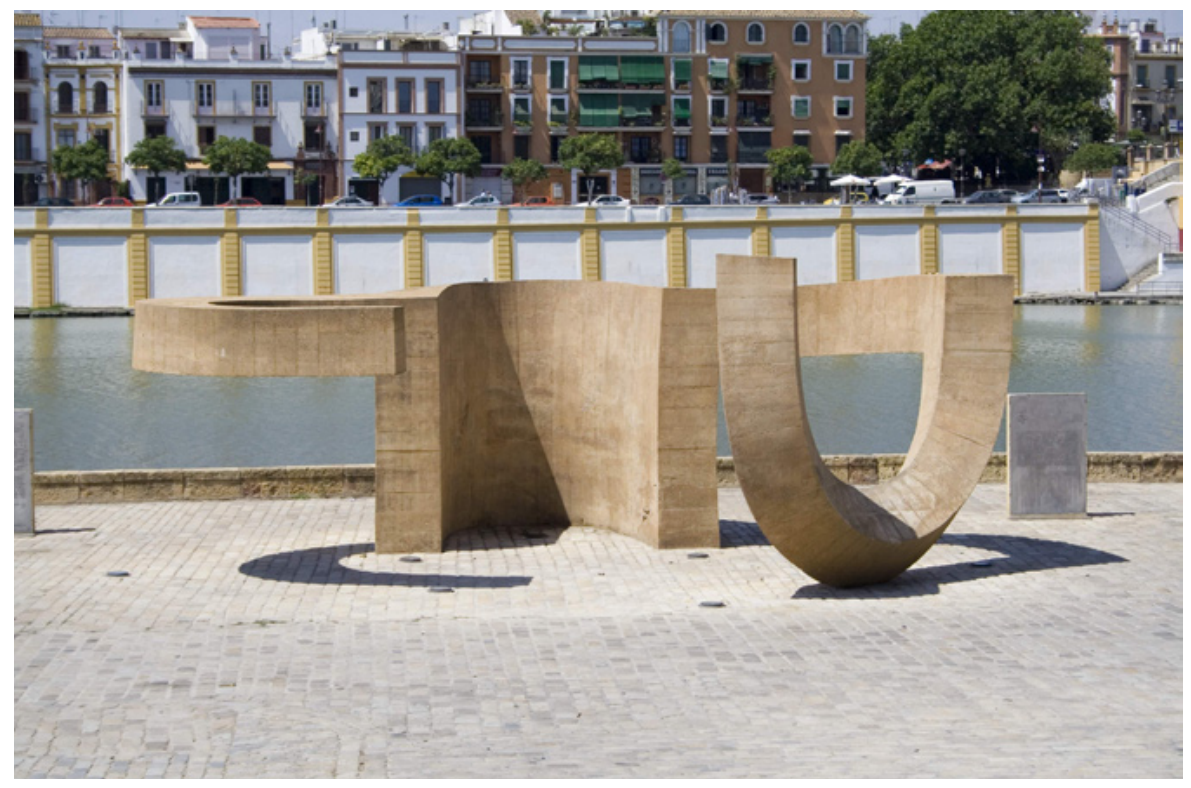

Eduardo Chillida. Monumento a la Tolerancia. 1992. Sevilla

| foto Fondo Gráfico IAPH (Luis F. Martínez Montiel)

Pese a que el trabajo se basa fundamentalmente en un análisis colectivo del mobiliario urbano, cada uno de los elementos más significativos quedará recogido en otra ficha con una serie de campos agrupados por módulos:

$>$ Identificación

$>$ Localización

$>$ Características físicas

$>$ Indicadores de valoración

$>$ Fuentes de información

"Identificación" recoge el título, los autores, los datos cronológicos y su clasificación dentro de una categoría o línea argumental.

En "Localización" se anotará la dirección en la que se encuentra el monumento así como una imagen, obtenida de Google Earth, en la que el propio bien queda marcado singularmente dentro del polígono de influencia que cada uno de los objetos seleccionados presenta. Posteriormente se georreferencia siguiendo el estándar establecido para Andalucía y habitual en otros trabajos del propio IAPH. Asimismo, se acompañará de dos o más imágenes, seleccionadas entre las instantáneas tomadas durante la fase de campo, que reflejen y sitúen el monumento individualmente y en su relación con el espacio que lo rodea.

El tercer módulo, "Características físicas", cuenta con una serie de campos en los que se pretende recoger técnicas, materiales, dimensiones, descripción, estado de conservación y otra serie de observaciones. 


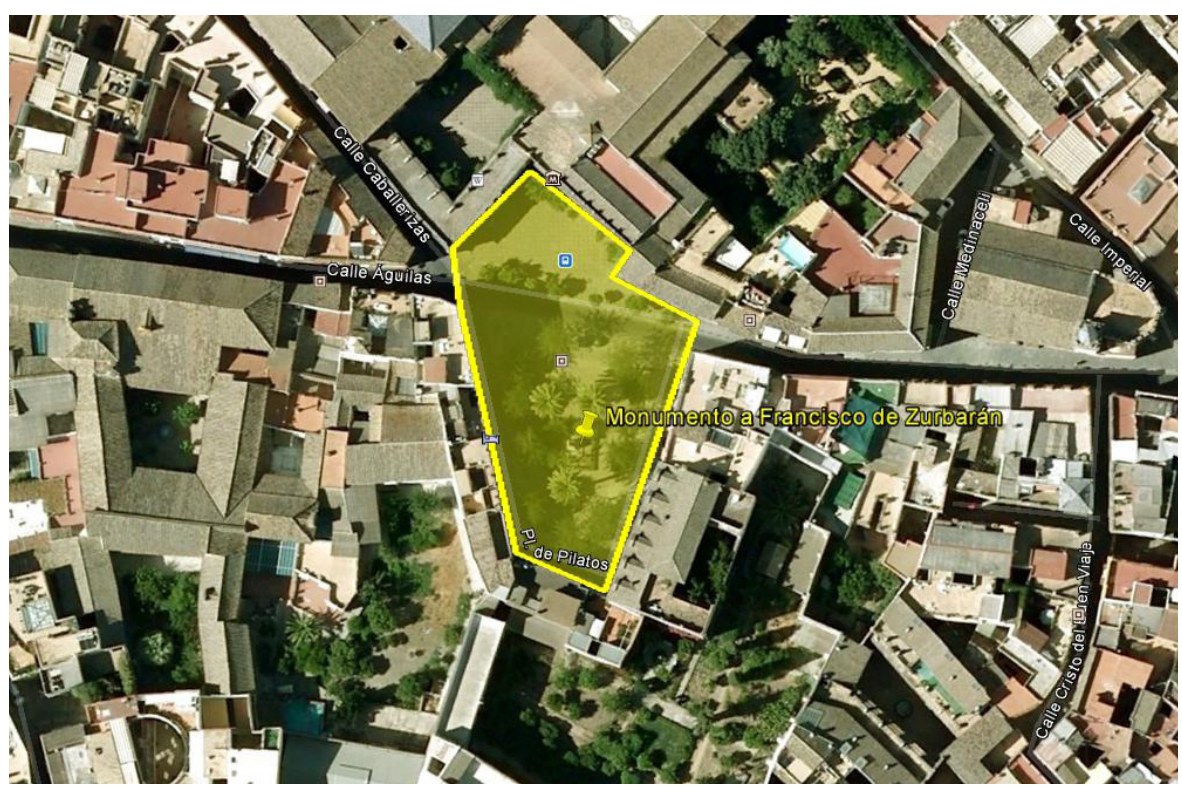

Aurelio Cabrera Gallardo. Monumento a Francisco de Zurbarán. 1929. Sevilla. Vista aérea y plano de localización (Google Earth)

El cuarto módulo, quizás el más importante y probablemente el menos usado en este tipo de trabajos hasta el momento, se dedica a los "Indicadores de valoración", que se analizan más adelante.

Finalmente, se recogerán las "Fuentes de información" utilizadas en cada uno de los objetos.

De gran importancia para los estudios de síntesis finales será la georreferenciación de los muebles. Como herramienta se propone utilizar el soporte informático suministrado por Google Earth, añadiéndole la información recogida en el trabajo de campo sobre el espacio en que la pieza queda integrada y que de alguna forma se relaciona directamente con ésta. Esta información quedará recogida en la misma herramienta informática mediante el trazado de polígonos que visualizan el área de relaciones mutuas. Estos contornos definidos posibilitan estudios de densidad y distribución del mobiliario urbano monumental diferenciado según las distintas categorías.

\section{Indicadores (ver Criterios) de valoración}

Se han definido una serie de indicadores de valoración divididos en tres tipos claramente diferenciados aunque en ocasiones íntimamente ligados unos a otros. Su conocimiento previo al acercamiento de los elementos estudiados posibilita no sólo análisis individuales de cada uno de los monumentos, sino también la obtención de análisis globales por tipologías. Los indicadores con los que se ha experimentado hasta el momento son: 


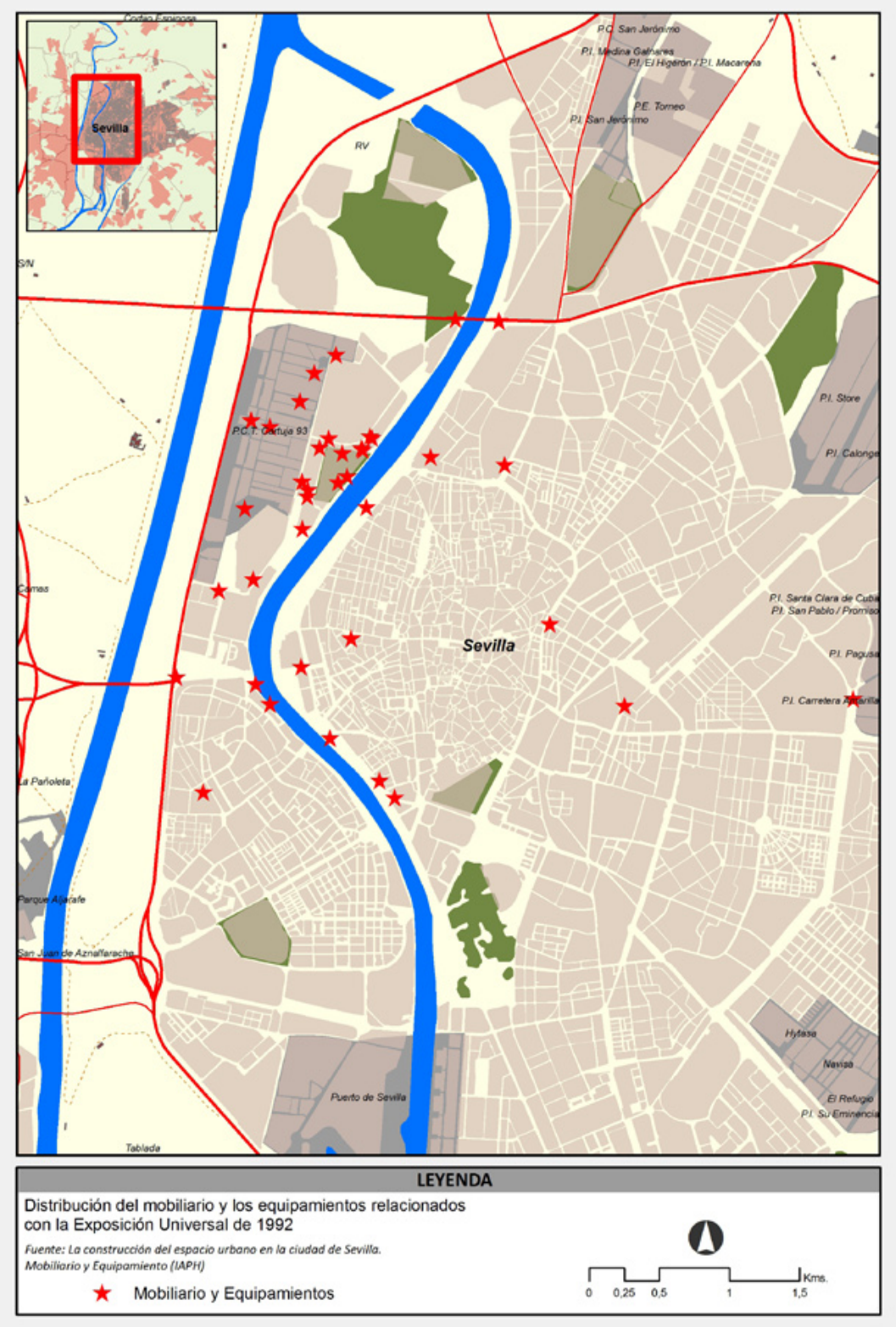

$>$ Valoración simbólica y significación cultural

$>$ Valoración formal

$>$ Valoración espacial

En cada uno de ellos se han valorado otra serie de variables que en conjunto van a permitir el conocimiento global de cada uno de los monumentos. En cualquier caso la puntuación -siempre de 1 a 5, siendo 1 el valor mínimo y 


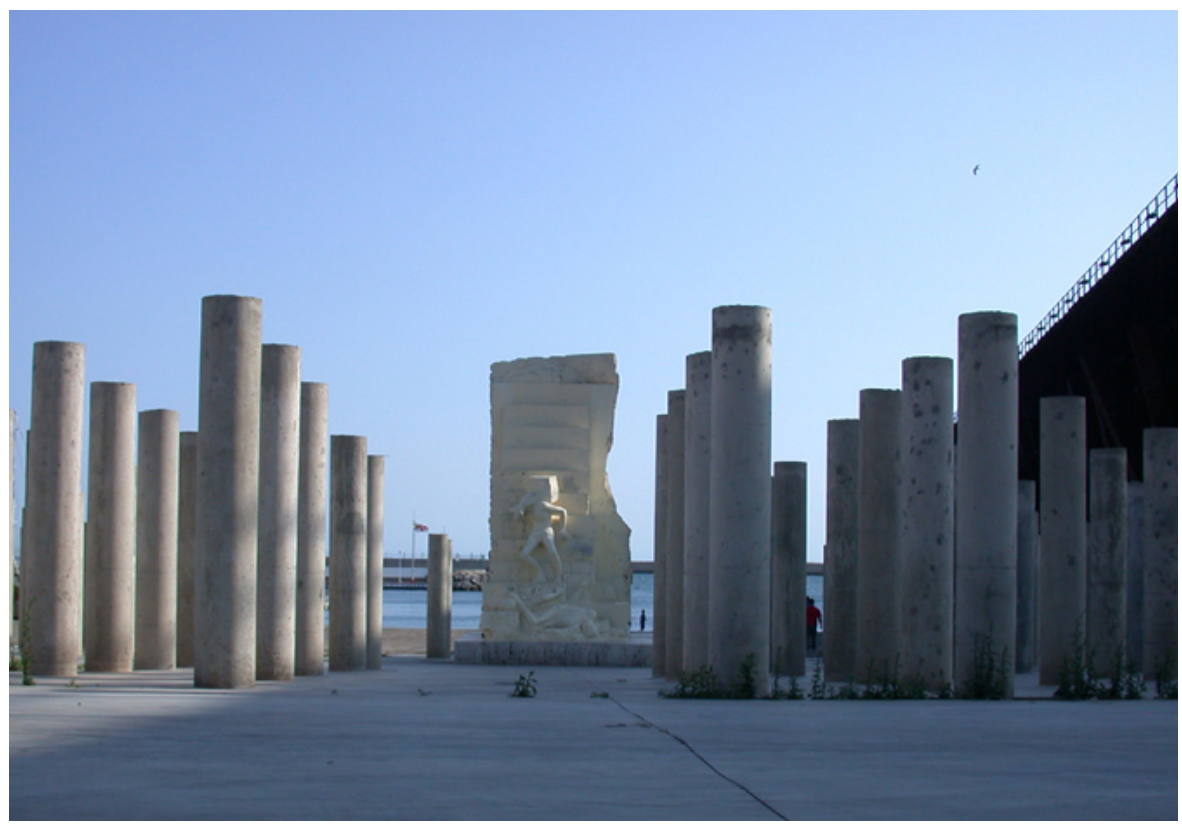

Ma Ángeles Lázaro Gil. Monumento a las víctimas de los nazis (o a la Tolerancia). 1999. Almería | foto Fondo Gráfico IAPH (Gloria Espinosa Spínola)

5 el máximo- no evalúa la bondad o maldad de la obra, sino la escala valorativa de ésta dentro de un conjunto. Constituye más bien un indicador de una serie de valores generales que actúan sobre el monumento, su emplazamiento y otra serie de cuestiones, como se verá más adelante. Por tanto, no resulta extraño que en muchos casos sorprenda la excesiva o la escasa valoración del monumento. Algunos de los indicadores pueden elevar esta puntuación sin que ello quiera decir que la pieza aisladamente merezca más alta valoración. La adecuación de la escala al entorno o su integridad pueden actuar como potenciadores de la puntuación final, mientras que su dudosa relación simbólico-espacial o la mala orientación pueden bajarla. No se debe, por tanto, olvidar que estos indicadores van más allá de la simple valoración objetual del monumento y que sobre todo establecen una visión más general de éste y del espacio en que se ubica. Evidentemente, puede ocurrir también en sentido contrario y que algunas obras de gran calidad vean mermada su valoración debido al efecto general que la baja puntuación en algunos indicadores produce. Todos estos factores deberán ser tenidos en cuenta finalmente cuando se formulen las diferentes propuestas.

\section{Valoración simbólica y significación cultural}

\subsection{Relación simbólico-espacial}

Pretende solventar la cuestión de la relación espacial entre el motivo representado y el espacio en el que se ubica. En no pocas ocasiones los monumentos aparecen desubicados, bien por el paso del tiempo y el posible olvido que ello conlleva y los traslados sin excesiva valoración, o simplemente por 
una mala elección desde el principio. Es frecuente encontrarlos distribuidos exclusivamente por un mal entendido sentido estético, que lo desliga en muchas ocasiones de su propuesta inicial.

\subsection{Trascendencia histórica del monumento}

Se intenta analizar la importancia que el motivo o el personaje representado tuvo en el momento de su creación. Se han tenido en cuenta principios locales, regionales, nacionales o internacionales. Asimismo se valora en este apartado la trascendencia de la propia obra. En este capítulo, de fuerte componente subjetiva, se debe intentar huir de los estereotipos o tópicos que acompañan a la mayoría de estas obras. Pese a ello, no hay que olvidar que en este trabajo los factores subjetivos son necesarios por la escasez de métodos objetivos de acercamiento. Es por tanto obligatorio asumir que, como individuos de una sociedad contemporánea, se está sujeto a determinados criterios y características que deben ser aplicados en los análisis de modo homogéneo, en la medida de lo posible.

1.3. Importancia del o los creadores de la obra

También se ha considerado como un valor analizable la mayor o menor trascendencia del autor, en función de su reconocimiento local, regional, nacional o internacional. Es este, de nuevo, un factor muy subjetivo y por tanto hay que proceder de forma muy cuidadosa. La aspiración de objetividad, sin renunciar al contenido crítico que toda valoración conlleva, ha planteado usar como un posible medidor neutral el número de resultados que devuelve Google tras una búsqueda por el nombre del artista. Este método no elimina ese factor crítico que debe ser realizado lo más objetivamente posible y que puede llevar a la necesidad de ponderar determinados resultados. Los criterios seguidos para evaluar las puntuaciones han sido: hasta 500 resultados (1), de 501 a 1.000 (2), de 1.001 a 5.000 (3), de 5.001 a 10.000 (4) y más de $10.000(5)$.

\section{Valoración formal}

\subsection{Valoración artístico/estética}

Se trata de la adecuación del monumento a los principios artísticos y estéticos que el criterio histórico ha considerado como básicos del periodo en el que fue creado.

\subsection{Adecuación de las texturas, materiales y técnicas}

Las técnicas, los materiales y las texturas son indicadores de la relación acertada del monumento con el espacio sobre el que actúa su influencia. Asimismo en esta variable se juzga también la adecuación de estos elementos a la funcionalidad concreta de cada monumento, ya sea la ornamental o la documental. Una clave que puede servir como medidor en este apartado es la relación entre el monumento en sí y el pedestal sobre el cual se levanta. 


\subsection{Nivel de integridad del monumento}

En muchos casos los monumentos, aquejados de ataques vandálicos o simplemente deteriorados por la acción del tiempo, han ido perdiendo la capacidad de evocar los principios para los que fueron creados. La desaparición de algunos elementos o su mutilación conducen a lecturas erróneas en todos los sentidos. Por tanto se considera que el nivel de integridad, en relación con el estado original, también condiciona la valoración.

\section{Valoración espacial}

La complejidad de la ubicación del monumento en el espacio y las complicadas relaciones que se establecen entre ambos definen en gran medida la trascendencia de éste.

Para su análisis se considera oportuno estudiar las siguientes variables:

\subsection{Valoración como generador de espacios}

Al margen de la relación simbólica que la obra presenta con el espacio que ocupa, puede considerarse como un valor cuantificable en sí mismo la ubicación espacial. Es decir, valorar su capacidad para generar espacios bien definidos y contribuir a la consolidación del paisaje urbano de la zona. De esta forma se puede apreciar desde su capacidad para convertirse en un foco gestor del espacio, hasta su especial irrelevancia para el área en el que se ubica. Este apartado refleja fielmente la trascendencia que los responsables de la ciudad otorgan a sus monumentos. La ubicación responde a una supuesta jerarquización del mismo dentro del conjunto de la ciudad y constituye un buen elemento para analizar las diferentes propuestas históricas en la gestión del espacio urbano de cada sociedad.

\subsection{Adecuación de la escala monumental al espacio}

La relación con las alturas y con la superficie del entorno en el que se ubica puede convertirse en un factor primordial para la trascendencia del monumento en la conformación de su entorno. A veces pasan desapercibidos por su falta de escala y en otros momentos se sobredimensionan produciendo una injerencia en el desarrollo de un espacio coherente. En muchos casos este hecho responde a la ya citada desubicación de los mismos y a los traslados poco estudiados y muy habituales.

3.3. Valoración de la adecuación del espacio inmediato al monumento Se pretende valorar las actuaciones próximas al monumento que intentan enmarcarlo en su ubicación. Con especial consideración al ornato vegetal, cerramientos, bancos, etc.

\subsection{Valoración posicionamiento y orientación}

Se valoran las perspectivas y campos visuales que se generan en torno al monumento. Fundamentalmente se pretende apreciar si el monumento en sí 


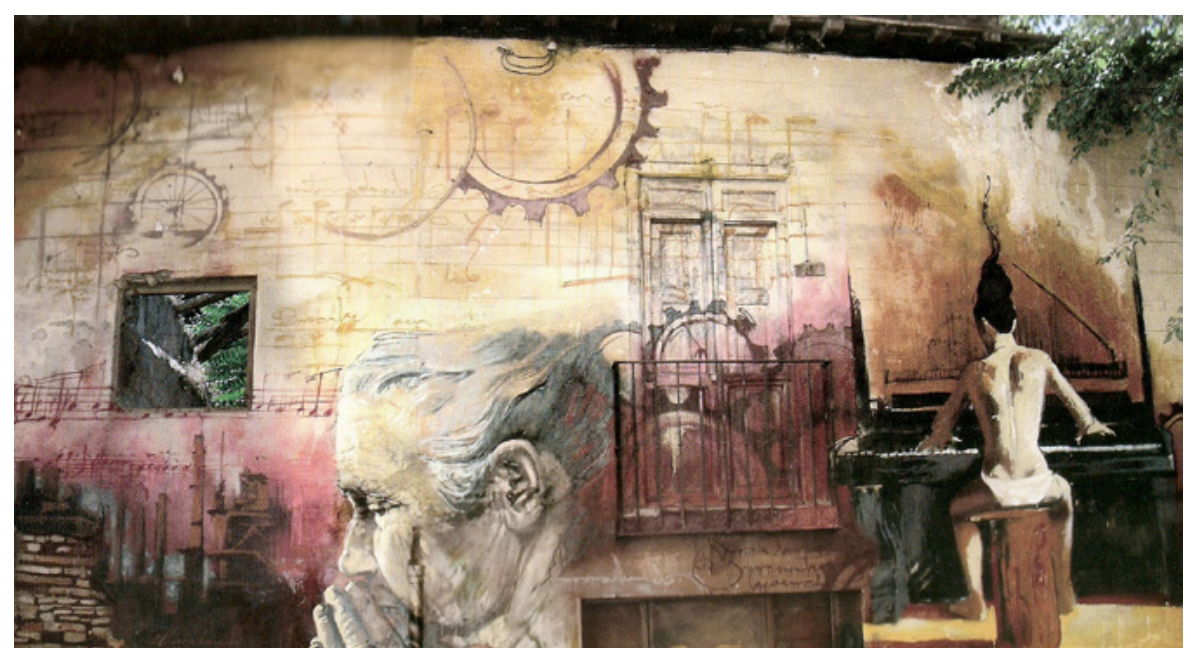

El Niño de las Pinturas. 2005. Granada. Cuesta de los Molinos

| foto Fondo Gráfico IAPH (María de la Encarnación Cambil Hernández)

presenta un adecuado posicionamiento y orientación en relación con el espacio que ocupa y con la visión y percepción que el ciudadano tiene de él.

\section{4. ${ }^{a}$ Fase. Registro, análisis, evaluación y establecimiento de criterios y propuestas de actuaciones sobre el mobiliario urbano}

A partir de las actividades y tareas expuestas, se elaborará el registro del patrimonio mueble urbano andaluz. Se plantearán una serie de recomendaciones genéricas con el objetivo de que sean tenidas en cuenta en las posibles actuaciones sobre el patrimonio urbano andaluz y extrapoladas a cualquier otra ciudad de similares características. Este análisis se pretende plantear en el ámbito de cada ciudad y a nivel territorial.

\section{5. ${ }^{\text {a }}$ Fase. Difusión y divulgación: el proyecto y sus resultados}

Se espera que con el desarrollo de los trabajos de investigación por parte de cada uno de los componentes del equipo en las ciudades andaluzas propuestas, se alcancen resultados publicables en revistas de prestigio relacionadas con el patrimonio histórico. En esas aportaciones no sólo se plantearán los distintos avances que se vayan produciendo en la investigación sobre el patrimonio mueble urbano, sino que también deberá darse a conocer la novedosa metodología propuesta y su aplicación, y habrá de contribuirse a que la comunidad científica conozca mejor y adquiera una mayor sensibilidad hacia este tipo de patrimonio.

La difusión y divulgación de los resultados del proyecto por distintos canales permitirá avanzar en el conocimiento y acceso a la información de este tipo de manifestaciones artísticas por parte de la ciudadanía. De esta forma 
aumentarán la sensibilidad y concienciación sobre la relevancia del patrimonio mueble urbano y la necesidad de su conservación, salvaguarda y -por qué no decirlo- de su justa valoración y retirada o traslado si procede.

La creación de una página web del proyecto posibilitará el conocimiento de sus objetivos, su descripción, la metodología empleada, la composición del equipo de investigación y las noticias y progresos más relevantes. Un enlace llevará al producto web resultado del registro de la información generada en el proyecto. Esta información podrá ser consultada por la ciudadanía.

Asimismo, se plantea la celebración de un seminario para la primavera de 2015, en el que se expondrán experiencias sobre el patrimonio mueble urbano, tanto nacional como internacional, poniendo especial énfasis en el localizado en el territorio andaluz. Asimismo, está prevista la publicación digital de las actas del seminario.

La culminación de este proyecto permitirá confeccionar una visión general del monumento público dentro del espacio urbano de la comunidad andaluza con lo que se contrastarán las actuaciones llevadas a cabo en él. Conservar un legado patrimonial, que en este caso es quizás uno de los más compartidos y por tanto más cercanos, constituye uno de los objetivos básicos de este proyecto, aunque no el único. El posicionamiento en la definición de la relación deseable entre los espacios urbanos y sus monumentos debe resultar prioritario. Se debe apostar por el "no vale cualquier cosa"; no todo monumento puede ni debe ubicarse en cualquier lugar por el simple hecho de que un colectivo más o menos amplio lo desee. El espacio urbano marca la vida de la ciudadanía, por lo que se debe ser extremadamente delicado con su uso monumental. Además hay que recordar que los criterios artísticos se encuentran regidos por principios que no siempre, afortunadamente, coinciden con los valores estéticos. Hay muchas manifestaciones en las que el dieciochesco concepto del "gusto", no debe ser el único valor; de lo contrario, perderíamos creaciones importantísimas no regidas exclusivamente por la belleza. Con este proyecto se puede contribuir a desvelar intereses espurios que, en más de una ocasión, provocan retrocesos en la consideración monumental de nuestras ciudades.

Como se decía, conservar y salvaguardar nuestro patrimonio monumental urbano es uno de los objetivos de este trabajo. Esta intención exige no permanecer ajenos a todas y cada una de las actuaciones que los gobernantes realizan sobre él, sino a cuestionarlas cada vez que se cometan errores y a valorarlas, resaltarlas y defenderlas cuando se promuevan aquellas que respondan al interés de toda la sociedad, y no al de unos pocos más o menos cercanos a quienes en cada momento gobiernan. La ciudad, como se decía al principio, es el fruto del pensamiento de todos y por tanto debe ser, en relación al trabajo que se comenta, gestionada con la más amplia de la miras. 


\section{BIBLIOGRAFÍA}

- BEBIANO, P. M. (1995) Mobiliario Urbano de Lisboa. 1838-1938. Dissertação de Mestrado. Lisboa: Universidade Nova, 1995

- BOYER, A.; ROJAT-LEFEBVRE, E. (1994)Aménagerles espaces publics: le mobilier urbain. Paris: Le Moniteur, 1994

- ESPIAU EIZAgUIRRE, M. (1983) El Monumento Público en Sevilla. Sevilla: Excmo. Ayuntamiento, 1983

- GARCÍA GUARDIA, G. (2012) Agua, mármol y bronce: ornato, símbolos y rumores por las calles de Granada. Granada: Caja General de Ahorros de Granada, Obra Social, 2002

- Guía del paisaje cultural de la Ensenada de Bolonia. Cádiz: Avance Sevilla: Consejería de Cultura, 2004 (PH cuadernos; 16)

- HERAS ESTEBAN, E. de las (2003) La escultura pública en Valencia. Estudio y catálogo, Valencia: Universitat de Valencia, 2003

- LAFITA GoRdillo, T. (1998) Sevilla Turística y Cultural. Fuentes y Monumentos Públicos. Sevilla: Prensa Española, 1998

- LOZANO BARTOLOZZI, M.M. (1988) Escultura pública y monumentos conmemorativos en Cáceres. Cáceres: Universidad de Extremadura, 1988

- El PAISAJE histórico urbano en las Ciudades Patrimonio Mundial: Indicadores para su conservación y gestión II. Criterios, metodología y estudios aplicados. Sevilla: Consejería de Cultura, 2009

- REYERO, C. (1999) La escultura conmemorativa en España: la edad de oro del monumento público, 18201914. Madrid: Cátedra, 1999

- RODRIGO CÁMARA, J.M.; DÍAZ IGLESIAS, J.M.; FERNÁNDEZ CACHO, S. et ál. (2012) Registro de paisajes de interés cultural de Andalucía. Criterios y metodología. PH: Boletín del IAPH, n. ${ }^{\circ} 81,2012$, pp. 64-75

- SÁNCHEZ LÓPEZ, J.A. (2007) Paseos por la escultura pública de Málaga. Málaga: Universidad de Málaga, 2007

- SÁNCHEZ LÓPEZ, J.A. (2005) La voz de las estatuas: escultura, arte público y paisajes urbanos de Málaga. Málaga: Universidad de Málaga, 2005

- SEgARRA LAGUNES, T. (2012) Mobiliario Urbano: historiay proyectos. Granada: Universidad de Granada, 2012

- SEIXAS, J.P.R. (1993) O Mobiliário urbano na cidade de Lisboa. Lisboa: s.n, 1993 (Tese mestr. Geografia Humana e Planeamento Regional e Local, Univ. Lisboa, 1993)

- SUBIRACHS I BURGAYA, J. (1989) L'Escultura conmemorativa a Barcelona (1936-1986). Barcelona: Amelia Romero editora, 1989 